\title{
Uneventful Disappearance of a Large Left Atrial Ball Thrombus with Enoxaparin in a Patient with Mitral Stenosis Associated with Pregnancy
}

\author{
Jorge Ernesto Martínez Espínola, María Isabel Acosta Colmán and Osmar Antonio Centurión*
}

Cardiology Department, Hospital de Clínicas, Asunción National University, Division of Electrophysiology and Arrhythmias. Cardiovascular Institute. Sanatorio Migone-Battilana. Asunción, Paraguay

\begin{abstract}
An atrial thrombus is a relatively common echocardiographic finding in patients with mitral valve stenosis (MVS) and atrial fibrillation (AF). However, a "ball thrombus" or floating thrombus in the left atrium is a rare and specific entity associated with MVS. A 24-year-old woman with rheumatic MVS presented with complaints of progressive dyspnea and inferior limbs edema that began 23 days earlier after a caesarean operation for stillbirth carried out at 8 months of pregnancy. At the time of hospitalization, she was in New York Heart Association functional class III and the ECG showed sinus rhythm. Transthoracic color-flow Doppler echocardiography revealed a thick, stenotic mitral valve with a valvular area of $0.9 \mathrm{~cm}^{2}$, and an echogenic large left-atrial mass diagnosed as a free-floating left-atrial thrombus that was corroborated by transesophageal echocardiography. She refused surgery and was treated medically, and low molecular weight heparin (LMWH) (enoxaparin $80 \mathrm{mg} / 12 \mathrm{~h}$ ) was given for 14 days and was discharged uneventfully on coumarin. Two days before discharge, a transthoracic and transesophageal ecocardiography showed disappearance of the ball thrombus uneventfully leaving spontaneous echo contrast inside the left atrium. To the best of our knowledge, this is the first case showing disappearance of a giant left atrial ball thrombus with LMWH treatment in a patient with severe MVS during sinus rhythm associated with pregnancy.
\end{abstract}

Keywords: Left atrial ball thrombus, Pregnancy, Mitral stenosis, Enoxaparin.

\section{INTRODUCTION}

Although a free-floating ball thrombus is a rare complication of mitral valve stenosis (MVS) [1-3], it is very important because of its potentially fatal effects. The term "ball thrombus" was first utilized by Word in 1814 when he described the autopsy of a young woman with rheumatic MVS and syncope with fainting spells occurring 3 to 4 times a day [2]. A floating ball thrombus is an uncommon finding in 1 out of 3000 autopsies [1].

The ball thrombus can produce sudden circulatory arrest and syncope by obstructing the mitral orifice, or can cause severe cerebral or peripheral thromboembolic events [3-5]. The clinical course could also be asymptomatic, and the diagnosis made as an ecocardiographic casual finding [5]. The incidence of atrial thrombus in young patients under 35 years of age with MVS and without atrial fibrillation (AF) is only $2 \%$. However, in the presence of AF the incidence is $6 \%$, and it goes up to $12 \%$ in patients over 35 years of age [6]. Pregnancy increases the risk of thromboembolic events in patients with MVS even in the absence of AF [4, 7]. It is known that pregnancy increases coagulation factors, and platelet adhesion, and decreases fibrinolysis setting the background for developing venous thrombi [7].

*Address correspondence to this author at the Department of Cardiology, Hospital de Clínicas, Asunción National University, Trejo y Sanabria 1657, Sajonia, Asunción, Paraguay; Tel/Fax: 595-21-421423;

E-mail: osmarcenturion@hotmail.com
We describe an unusual case of a young woman with MVS who developed an uneventful ball thrombus after a caesarean section in the absence of AF. To the best of our knowledge, this is the first case showing disappearance of a giant left atrial ball thrombus with low molecular weigth heparin (LMWH) treatment in a patient with severe MVS during sinus rhythm associated with pregnancy.

\section{CASE REPORT}

A 24-year-old woman with rheumatic MVS presented with complaints of progressive dyspnea and inferior limbs edema that began 23 days earlier after a caesarean section for stillbirth carried out at 8 months of pregnancy. At the time of hospitalization, she was in New York Heart Association (NYHA) functional class III in sinus rhythm; her blood pressure was $110 / 70 \mathrm{mmHg}$. Auscultation revealed a loud $1 \mathrm{st}$ heart sound, a constant early opening snap, and a middiastolic murmur in the mitral focus. She had mild lung congestion and ascitis, hepatomegaly and inferior limbs edema was present. The chest radiograph showed cardiomegaly, left-atrial enlargement, dilated pulmonary arteries and pulmonary venous congestion. The ECG showed sinus rhythm. Transthoracic color-flow Doppler echocardiography revealed a thick, stenotic mitral valve with a valvular area of $0.9 \mathrm{~cm}^{2}$, and an echogenic large left-atrial mass diagnosed as a freefloating left-atrial thrombus $(24 \times 19 \mathrm{~mm})$ that was corroborated by transesophageal echocardiography Fig. (1). She also had tricuspid regurgitation with a pressure gradient of 55 $\mathrm{mmHg}$ and mild pericardial effusion. Blood tests were normal except for hemoglobin $9.1 \mathrm{~g} / \mathrm{dL}$. 


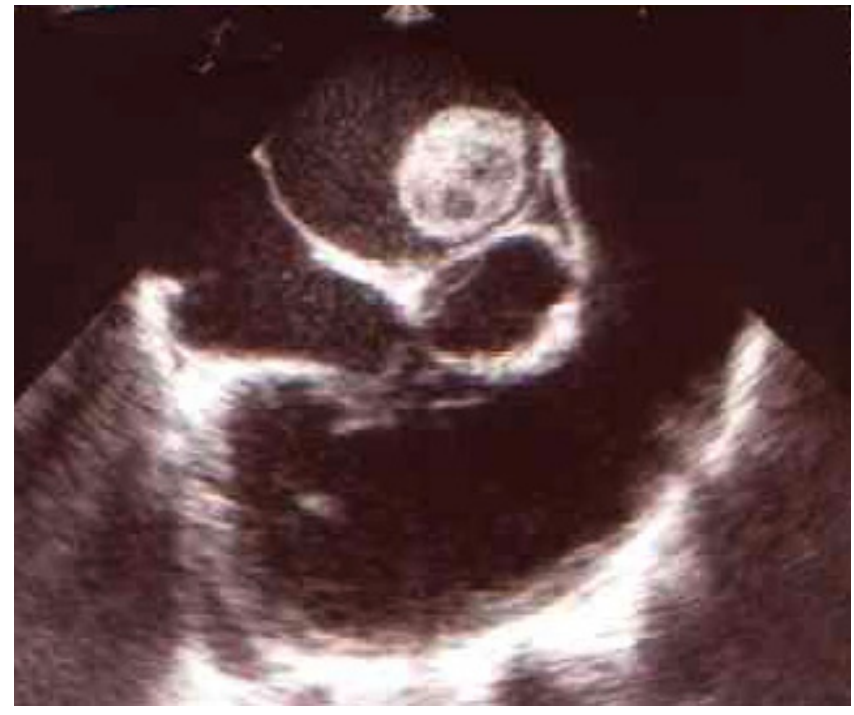

Fig. (1). Transesophageal color-flow Doppler echocardiography showing an echogenic large left-atrial mass diagnosed as a freefloating left-atrial thrombus ( 24 x $19 \mathrm{~mm})$.

She refused surgery and was treated medically with diuretics, angiotensin converting enzime (ACE) inhibitors, and LMWH (enoxaparin $80 \mathrm{mg} / 12 \mathrm{~h}$ ) for 14 days and was discharged uneventfully on coumarin. Two days before discharge, a transthoracic and transesophageal ecocardiography showed disappearance of the ball thrombus leaving spontaneous echo contrast inside the left atrium Fig. (2).

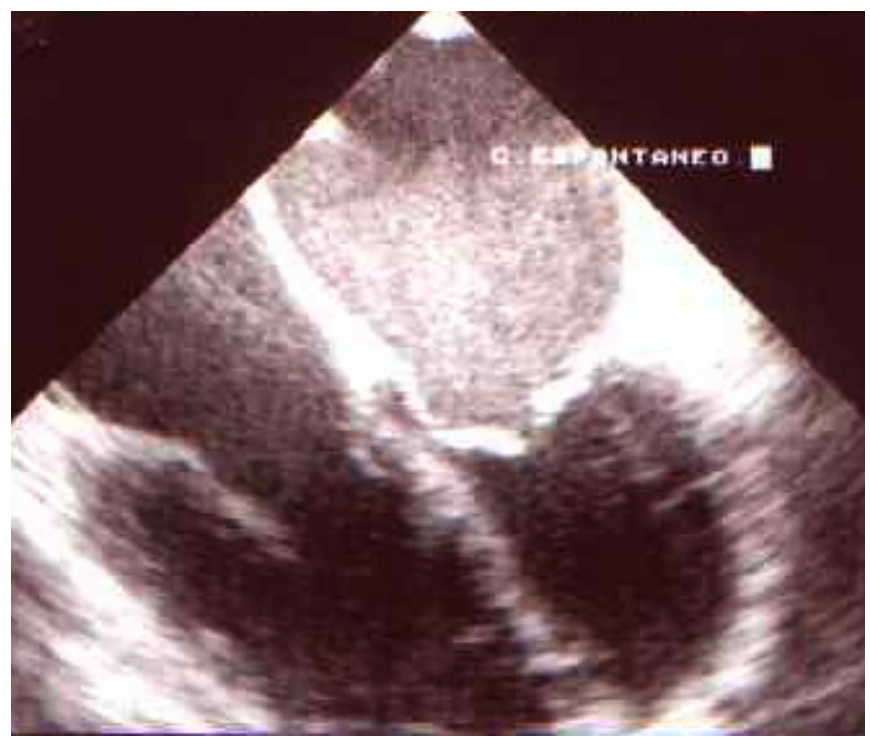

Fig. (2). Transesophageal color-flow Doppler echocardiography showing disappearance of the ball thrombus with LMWH treatment in an uneventful manner. There is spontaneous echo contrast remaining inside the left atrium.

\section{DISCUSSION}

The clinical diagnosis of a ball thrombus is difficult, however, the presence of left-atrial ball thrombus should be suspected if patients with MVS and AF have intermittent or changing murmurs, emboli or syncope [3]. A ball thrombus is thought to originate as a small mural thrombus, usually attached to the interatrial septum, and rarely in the left-atrial appendage. The thrombus gradually thickens and enlarges to form a projecting mass that remains attached to the atrial wall by a pedicle. Later on, as the thrombus enlarges, the pedicle gets longer and thiner until eventually the thrombus separates from the atrial wall [8]. Thereafter, as the thrombus spins freely in the atrium, it acquires its characteristic smooth, polished appearance.

$\mathrm{AF}$ is a common arrhythmia and a major cause of morbidity and mortality from stroke and thromboembolism, usually due to embolization of thrombus formed within the fibrillating left atrium and its appendage [6]. AF is associated with a prothrombotic or hypercoagulable state, with evidence of abnormal hemostasis, endothelial damage/dysfunction, and platelet activation [6]. However, our patient did not have AF and did not complain of palpitations. She was in sinus rhythm throughout the hospitalization. Although, we can not rule out the presence of AF before hospitalization, the clinical course suggests that she did not have a tachyarrhythmia. Besides, there is a known correlation between pregnancy and thrombus formation in patients with MVS despite of being in sinus rhythm [3]. There is a hipercoagulability state during pregnancy, hence, pregnant patients with MVS are prone to develop thromboembolic complications $[3,7]$. This increase in the incidence of thromboembolism is greatly related to coagulation changes during gestation, which include an increase in levels of factors I, II, VII, VIII, IX, and X after the first trimester of pregnancy and subsequent increase in factors V, VII, VIII, and X after the delivery [7]. These changes in coagulation during pregnancy are associated with a significant rise in the incidence of venous thrombosis, and may further enhance the already increased risk of thromboembolism in women with MVS.

Cardiac thrombus causing a stroke is found in the left atrium in $45 \%$ to $65 \%$ [3]. The incidence of thrombus in the left atrial appendage is $30 \%$ to $50 \%$ of the total number of left atrial thrombus, and these are very difficult to diagnose with transthroracic echocardiography [8]. Our pregnant patient with MVS developed a left atrial ball thrombus that was diagnosed in the peri-partum period after a caesarean section. The diagnosis was made by transthoracic echocardiography and corroborated by transesophageal echocardiography. Once the diagnosis of a ball thrombus is made, surgery is the gold standard theraphy with a $90 \%$ long-term survival, since anticoagulation or thrombolisis may cause embolization. Given the unpredictability of other modalities for treating free-floating left atrial thrombi and the obvious inevitability of eventual catastrophic complications, prompt surgical removal is the therapy of choice. Therefore, surgical extraction of the ball thrombus with mitral valve replacement should be indicated in MVS patients. However, since our patient refused surgery, she was treated medically with diuretics, angiotensin converting enzime (ACE) inhibitors, and enoxaparin $80 \mathrm{mg} / 12 \mathrm{~h}$ for 14 days and was discharged uneventfully on coumarin. Two days before discharge, a transthoracic and transesophageal echocardiography showed an uneventful disappearance of the ball thrombus leaving spontaneous echo contrast inside the left atrium. LMWH were utilized in other types of intracardiac thrombus [9], 
however, to the best of our knowledge, this is the first case showing dissappearance of a giant left atrial ball thrombus with LMWH treatment in a patient with severe MVS during sinus rhythm associated with pregnancy.

\section{CONFLICT OF INTEREST}

None declared.

\section{ACKNOWLEDGEMENTS}

None declared.

\section{REFERENCES}

[1] Lie JT, Entman ML. "Hole-in-one" sudden death: mitral stenosis and left atrial ball thrombus. Am Heart J 1976; 91: 798-804.

[2] Wood W. Letter enclosing the history and dissection of a case in which a foreign body was found within the heart. Edinburg Med J 1814; 10 : 50 .
[3] Wrisley D, Giambartolomei A, Lee I, Brownlee W. Left atrial ball thrombus: review of clinical and echocardiographic manifestations with suggestions for management. Am Heart J 1991; 121: 1784-90.

[4] Afshan H, Mohammed W, Akhter D, Fahed B. Left atrial thrombosis in pregnant woman with mitral stenosis and sinus rhythm. Am J Obst Gynecol 2005; 193: 501-4

[5] Vitale N, Agnino A, Serena D. Asymptomatic large left-atrial ball thrombus secondary to mitral stenosis. Tex Heart Inst J 1997; 24: 376-8.

[6] Rowe JC, Bland EF, Sprague HB. The course of mitral stenosis without surgery: ten and twenty year's perspectives. Ann Intern Med 1960; 52: 741-9.

[7] Danzell JD. Pregnancy and pre-existing heart disease. J La State Med Soc 1998; 150: 97.

[8] Hameed AB, Wani OR, Karaalp IS, et al. Valvular disease in pregnancy: Effects on maternal and fetal outcome. Circulation 1999; 100: $1-148$.

[9] Mallory R, Balcezak T. Treatment of mobile left ventricular thrombus with low molecular weight heparin. N Engl J Med 1999; 341: $1082-3$.

(C) Espínola et al.; Licensee Bentham Open.

This is an open access article licensed under the terms of the Creative Commons Attribution Non-Commercial License (http://creativecommons.org/licenses/ by-nc/3.0/) which permits unrestricted, non-commercial use, distribution and reproduction in any medium, provided the work is properly cited. 